\title{
Influence of inner structural parameters on the inner flow and outer spray characteristics for internal impinging nozzle
}

\author{
Zhijun WU1 ${ }^{1}$, Pengfei LENG ${ }^{1}$, Yu GAO ${ }^{1}$, Zongjie $\mathrm{HU}^{* 1}$, Wenbo ZHAO ${ }^{1}$, Yufeng WANG ${ }^{2}$, Wei \\ $\mathrm{XIE}^{1}$, Liguang $\mathrm{LI}^{1}$ \\ ${ }^{1}$ School of Automotive Studies, Tongji University, China \\ ${ }^{2}$ Jiangsu Riying Electronics Co., Ltd., China
}

\begin{abstract}
The impinging nozzle is widely used in the vehicle cleaning system because of the unique advantages in spray dispersion and fine droplet generation. The internal structures of the impinging nozzle can influence the internal flow pattern, which consequently determines the spray characteristics. In this paper, a numerical model simulating the internal flow field and the external spray field was proposed to study the influence of the impinging nozzle structures on the internal flow and external spray characteristics. The model was verified by experimental results including different spray sweep angles, sweep frequencies, and total flow rates under different pressures. As the impact angle increased from 80 degrees to 120 degrees, the spray sweeping angle and the total mass flow rate continuously decreased and the sweeping frequency reached a peak when the impact angle was 100 degrees. With the increase of the cavity exit width, the spray sweep angle and the total flow rate rose while the spray sweep frequency shifted down. When the shoulder radius is greater than $0.88 \mathrm{~mm}$, the spray characteristics are more sensitive to the shoulder radius. The influence mechanism of the structures on spray characteristics was analyzed according to the internal flow results.
\end{abstract}

\section{Keywords}

Impinging nozzle, impact angle, cavity exit width, shoulder radius, spray characteristics

\section{Introduction}

The internal impinging nozzle (also known as jet-interaction fluidic oscillator) is used as flow control actuator with air as the working fluid in the aerospace field recent years [1-3]. But the most common daily use of the impinging nozzle is as windshield washer fluid nozzle [4]. The performance of the nozzle is decided by the internal flow, which is affected by the inner structure of the nozzle. The basic principle of the impinging nozzle is the unsteady interaction of two jets within a cavity without feedback channels, that lead to an unsteady external jet [4]. Due to the special structure of the impact cavity, several periodic vortices are generated when the fluid respectively enter in the impact cavity through the two inlet paths forming two mutually impacting jets. These vortices drive the jets to switch periodically, forming a feedback mechanism without external interference, and causing periodic oscillation of the external jet. In order to investigate the details of the jet interaction behaviour, several researchers [5-7] used experimental and numerical approaches to visualize the internal flow field of the impinging nozzle. They found that the two jets collided at the center of the impact cavity, and vortices with opposite rotation directions were generated on both sides of the impact point. The shape of the vortices would change continuously during the oscillation process [5].

Some studies[6-8] focus on the effects of nozzle structures on spray characteristics. Tomac et al.[7] studied the impact of geometry and fluid selection on the oscillation characteristics of impinging nozzle. Bidadi et al. [6]established the numerical model and analysed the difference between the two-dimensional model and the three-dimensional model. Jhaveri et al.[8] found that the dimensional variation, material, and manufacturing process of the nozzle had 
significant influence on the performance of the nozzle. The variation of the geometry of the impact chamber and the width of the outlet can affect the oscillation frequency, and the driving pressures required by the nozzles with different geometry are also different.

In this study, a numerical model was established to investigate the effects of some key inner structural parameters on internal flow and spray characteristics.

\section{Numerical Method}

As shown in Figure 1 and Figure2, the impinging nozzle consists of the nozzle inlet, nozzle outlet, impact cavity, impact cavity exit, and two inlet paths. The intersection of the extended centerlines of the two inlet paths is defined as the impact point, and the angle of the extended centerlines is defined as the impact angle. The inner structure of the impinging nozzle is complex, containing many curved surfaces, which leads to the use of unstructured grids for the internal flow field simulation. The maximum grid size was set to $0.1 \mathrm{~mm}$ and the minimum was set to $0.04 \mathrm{~mm}$.

The hybrid mesh scheme was used in the external computational domain, which can get a better resolution for liquid phase distribution [10]. Unstructured tetrahedral grids were adopted close to the nozzle outlet, while structured hexahedral grids were adopted for the region far away from the nozzle outlet. In order to observe the complete spray pattern, the external computational domain was set to $250 \mathrm{~mm} \times 210 \mathrm{~mm} \times 70 \mathrm{~mm}$, as shown in Figure 3 . Combining the flow field mesh inside and outside the nozzle, we can get the entire computational domain.The origin of the coordinate system of the entire computational domain was located at the center of the nozzle outlet.

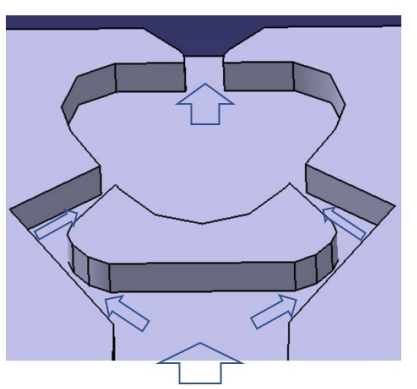

Figure1. The 3D shape of the nozzle.

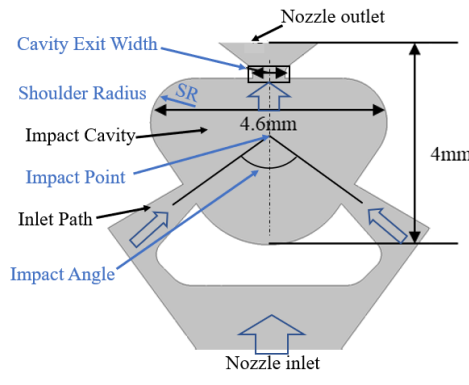

Figure 1. The definition of the nozzle structure.

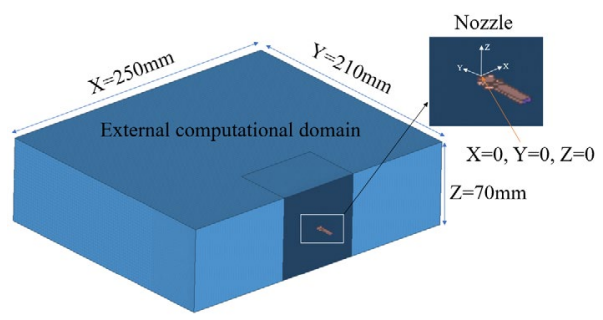

Figure 3. Flow field computational domain of internal impinging nozzle.

ANSYS-Fluent was used for this calculation, and water was selected as the working fluid. The VOF (Volume of Fraction) model is used to simulate the two-phase flow (air and water) by solving the momentum equation and the volume fraction of different components separately. Realizable k-epsilon model is selected for calculation to improve the computational stability and solution accuracy.

Table 1. The calculation solver settings.

\begin{tabular}{cc}
\hline Solver & Setting \\
\hline Scheme & SIMPLE \\
Gradient & Green-Gauss Node Based \\
Pressure & Body Force Weighted \\
Momentum & \\
Volume Fraction & Second Order Upwind \\
Turbulent Kinetic Energy & \\
Turbulent Disspation Rate & \\
\hline
\end{tabular}


The calculation solver settings are shown in Table1. The computational domain of the nozzle internal flow field is initialized with water, while the external computational domain is set with air at $0.1 \mathrm{MPa}$. The experimentally measured spray sweep frequency is $830 \mathrm{~Hz}$. Therefore, the calculation time step was set to $0.01 \mathrm{~ms}(10000 \mathrm{~Hz})$ to ensure the temporal accuracy. In order to observe a stable spray pattern in the external flow field, the maximum number of iterations for each calculation step was 20 and the total calculation step was set to 10000 .

In order to verify the credibility of the simulation model, the calculation results of spray sweep angle, spray sweep frequency, and total flow rate were compared with experimental results.

The water phase volume fraction distribution is used to depict the simulated spray morphology under different injection pressures and is compared with the experimental results obtained by high-speed Schllieren technique, as shown in Figure 4, and the spray sweep angle was marked. Slight difference of spray morphology under different injection pressures can be found in the results, and the experimental and simulation results are in good agreements. The maximum error of sweep angle results is $2.2^{\circ}$ and the relative error rate of the experimental value is only $4.7 \%$. The results also indicate that the injection pressure has almost no effect on the water phase volume fraction distribution.

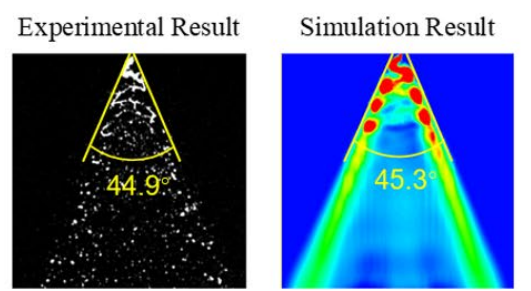

(a)Injection Pressure $=0.12 \mathrm{MPa}$

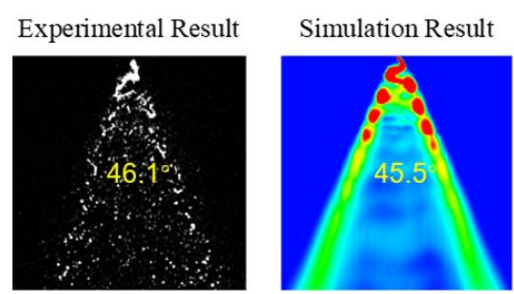

(c) Injection Pressure $=0.16 \mathrm{MPa}$

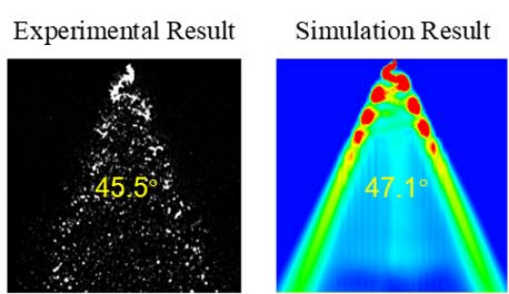

(e) Injection Pressure $=0.20 \mathrm{MPa}$

Figure 4. The experimental spray morphology and the numerical results of water phase volume fraction contour.

The monitoring point was set at the origin of the coordinate system in Figure 3 to obtain the flow velocity information. The spray sweep velocity is defined as the horizontal velocity along the sweep direction (along the $x$-direction in Figure 3). In the steady state, the changing trends of spray sweep velocity under different injection pressures are similar to sinusoid form, as shown in Figure 5. In order to eliminate the interference of time, the phase of the sweep velocity is defined instead of time to describe the sweep period in the subsequent analysis. The first point where the velocity changes from negative to positive is selected as the $0^{\circ}$ phase, and the first point where the velocity changes from positive to negative is set as $180^{\circ}$ phase. The spray sweep frequency of the simulation results can be obtained from Figure 5 .

Figure 6 shows the comparison of experimental results and simulation results of spray sweep frequency at different injection pressures. Take the simulation result of the average flow rate in a whole sweep period as the total flow rate of the nozzle. The comparison of total flow rate of the numerical and experimental results is shown in Figure 7. The sweep frequency and the total flow rate both increase with the increase of injection pressure. The maximum error of the total flow rate is $7.7 \%$, and the maximum error of sweep frequency is only $1.7 \%$, which indicates an excellent consistency between the numerical and experimental results.

Therefore, this model with high fidelity is credible for further studies. The influence of impinging nozzle structures, including the impact angle (IA), the cavity exit width (CEW), and the shoulder radius (SR), on internal flow and spray characteristics will be discussed in the next section. 


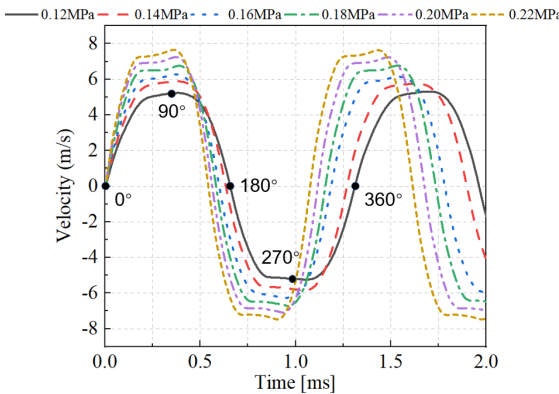

Figure 5. The spray sweep velocity under different injection pressures.

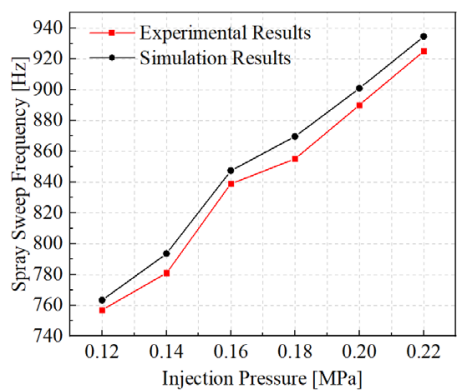

Figure 6. Relation between sweep frequency and injection pressure.

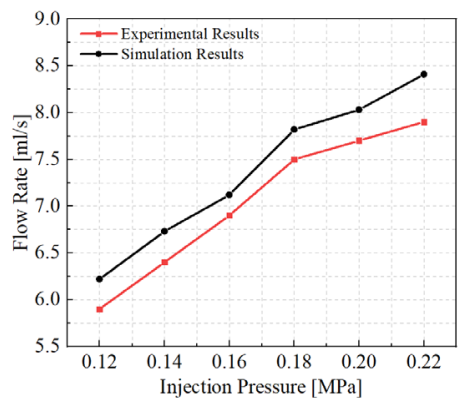

Figure 7. Relation between total flow rate and injection pressure.

\section{Results and Discussion Effect of Impact Angle}

The impact angle and the position of the impact point are shown in Figure 2. The position of the impact point is kept fixed when changing IA. The variation of spray sweep angle, sweep frequency and total flow rate with IA is shown in Figure 8 . When IA increases from $80^{\circ}$ to $120^{\circ}$, the spray sweep angle decreases from $94.56^{\circ}$ to $34.15^{\circ}$, and the total flow rate decreases from $7.34 \mathrm{~mL} / \mathrm{s}$ to $6.93 \mathrm{~mL} / \mathrm{s}$. When $\mathrm{IA}$ is less than $100^{\circ}$, the spray sweep angle is greatly affected by IA. With the increase of IA, the spray sweep frequency first increases and then decreases. When IA is $100^{\circ}$, the spray sweep frequency reaches the peak value. It can be seen that IA has a great influence on the sweep angle, followed by the frequency and the least influence on the total flow rate.

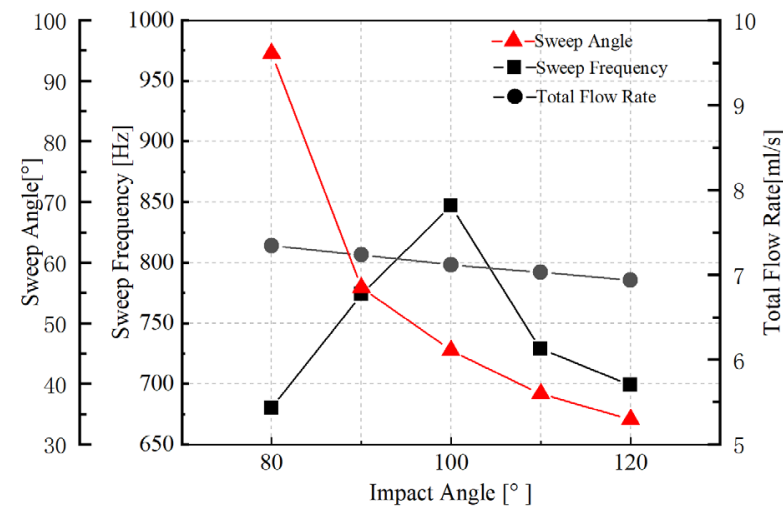

Figure 8. Variation law of spray sweep angle, sweep frequency and total flow rate with impact angle.

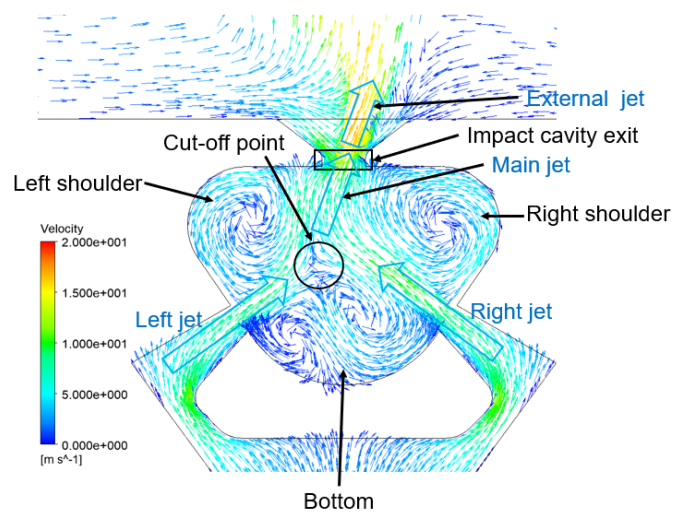

Figure 9. The names of some jets and nozzle structures.

The names of some key flow features and nozzle structures are shown in Figure 9. In the steady oscillating stage, the water phase volume fraction distribution of the external flow field, the velocity vector distribution, and the pressure distribution inside the nozzle with different IA at $90^{\circ}$ phase are shown in Figure 10. It can be seen from the velocity distribution of the internal flow field that the horizontal distance between the jet inlets on the left and right sides increases as IA increases, and the cut-off point gradually approaches the impact exit, which leads to a decrease in the working fluid of the left jet flowing into the main jet, and results in a decrease in the total flow rate of the nozzle. As the position of the cut-off point gets closer to the exit, more working fluid of the main jet can leave directly from the impact cavity exit, and less working fluid is obstructed by the left side of the impact cavity exit. As a result, the pressure difference between the left and right sides of the impact cavity is decreased and the spray sweep angle becomes smaller. As IA increases, the sweep distance of the main jet inside the 
nozzle became shorter, so the time of one sweep period is shortened, and the sweep frequency increases. But when IA continues to increase over $100^{\circ}$, although the spray sweep angle continues to decrease, the jet sweep velocity becomes slower due to the lower pressure difference between the left and right sides of the impact cavity, and the sweep frequency decreases.

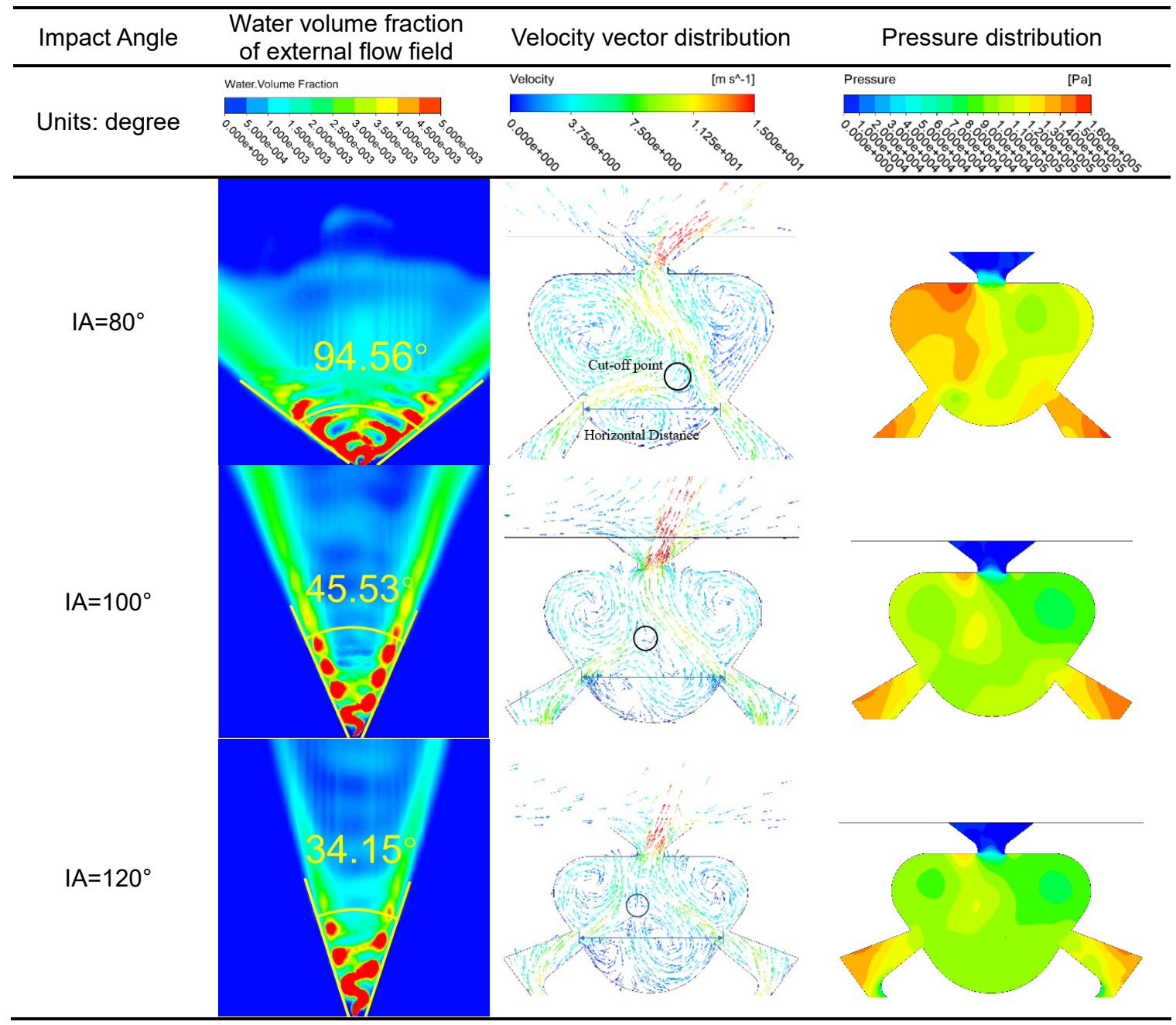

Figure 10. The water phase volume fraction distribution of the external flow field, internal flow field velocity vector distribution and pressure distribution of the nozzle with different IA at the $90^{\circ}$ phase.

\section{Effect of Cavity Exit Width}

The location of the impact cavity exit is shown in Figure 2. As the cavity exit width increases, the sweep angle is enlarged and the total flow rate is changing in an approximately linear pattern and both of them are affected obviously. Meanwhile, the sweep frequency is reduced from $955 \mathrm{~Hz}$ to $813 \mathrm{~Hz}$, as described in Figure 11. Compared with Figure 8, reducing CEW can significantly increase the sweep frequency.

Figure 13 shows that, even if CEW gets wider, the flow regime at the cavity shoulder and bottom is almost invariable, but the velocity of the main jet increases, which enhances the total flow rate and lowers the overall pressure inside the nozzle. As CEW increases, more fluid can flow out through a larger exit area, and the main jet near the cavity exit can reach further to the left as shown in Figure 13. Further deflection distance of the main jet makes the sweep frequency reduced. When CEW is $0.85 \mathrm{~mm}$, the deflected jet at the cavity exit can be more fully developed instead of being restricted by the right wall, and the high-pressure area at the 
left side of the cavity exit is closer to the exit causing the high pressure region inside the cavity exit to shift to the left. That is the reason why the sweep angle grows larger.

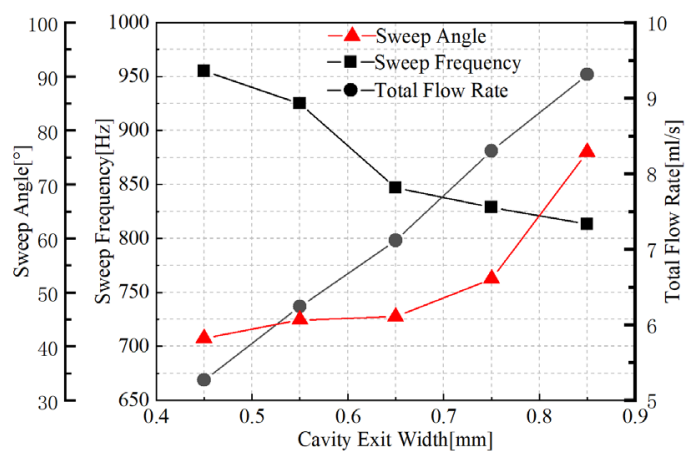

Figure 11. Variation law of spray sweep angle, sweep frequency and total flow rate with CEW.

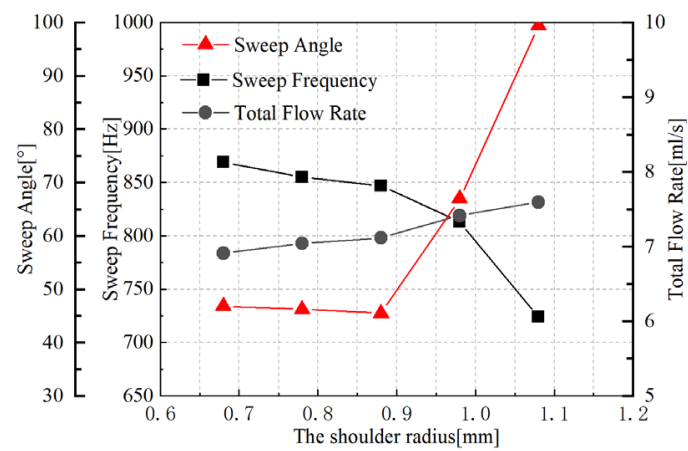

Figure 12. Variation law of spray sweep angle, sweep frequency and total flow rate with SR.

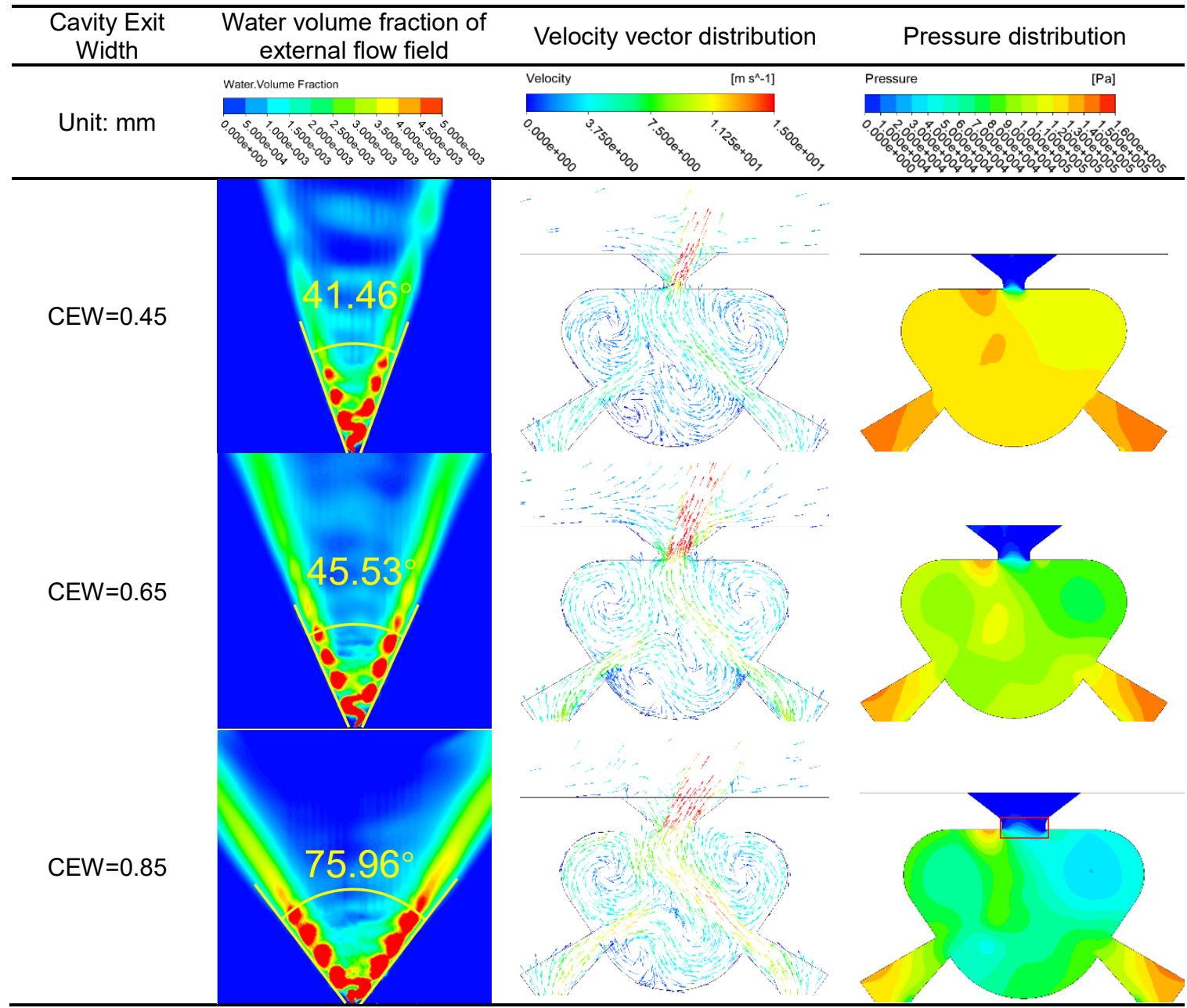

Figure 13. The water phase volume fraction distribution of the external flow field, internal flow field velocity vector distribution and pressure distribution of the nozzle with different CEW at the $90^{\circ}$ phase.

\section{Effect of Shoulder Radius}

The variation of the sweep angle, frequency, and the total flow rate with the shoulder radius is shown in Figure 12. As the shoulder radius increases from $0.68 \mathrm{~mm}$ to $0.88 \mathrm{~mm}$, the spray characteristics roughly keep unchanged. However, when SR is greater than $0.88 \mathrm{~mm}$, the 
sweep angle increases rapidly and the total flow rate slightly increases, while the sweep frequency is reduced by about $15 \%$.

As the SR increases, the volumes of the left and the right shoulders are reduced. As illustrated in Figure14, the velocity vector distribution and pressure distribution are basically same as those when SR increases from $0.68 \mathrm{~mm}$ to $0.88 \mathrm{~mm}$. This is because the decrease of the volume of the left and right shoulder does not affect the development of the vortex there. In the other words, when SR increases from $0.68 \mathrm{~mm}$ to $0.88 \mathrm{~mm}$, the volume is still large enough for the development of the vortex. But when SR is greater than $0.88 \mathrm{~mm}$, the vortex on the right shoulder cannot be fully developed causing the main jet to deflect to the right. Therefore, more water directly flows out through the exit leading to the increase of the total flow rate and the pressure drop in the nozzle, especially at the left of the cavity exit. Consequently, the pressure difference between the left and right sides decreases which causes the reduction of the jet sweep speed and the sweep frequency. Given that CEW is constant, the deflected jet makes the pressure at the left side inside the cavity exit increases. The pressure difference drives the jet to deflect further, so the sweep angle became bigger.

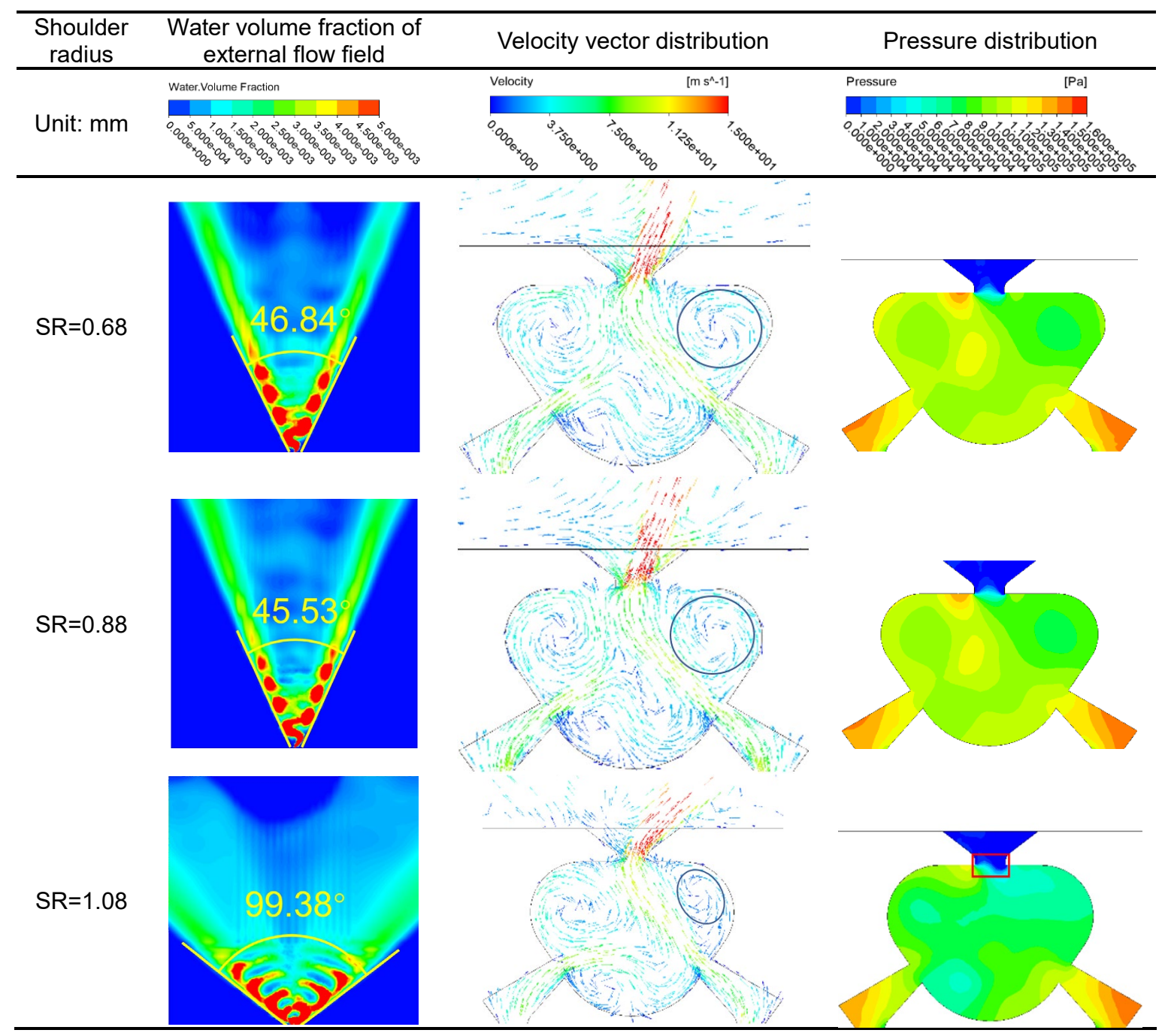

Figure 14. The water phase volume fraction distribution of the external flow field, internal flow field velocity vector distribution and pressure distribution of the nozzle with different $S R$ at the $90^{\circ}$ phase. 


\section{Conclusions}

In this paper, the influence of three key nozzle inner structure parameters, including the impact angle, the cavity exit width, and the shoulder radius on the spray characteristics is investigated by numerical simulation. The mechanism of their influences are revealed from the perspective of internal flow. The numerical results showed excellent consistency with the experimental data in terms of spray sweep angle, spray sweep frequency, and total flow rate. The main conclusions are as follows.

(1) As IA increases, the spray sweep angle decreases rapidly, the total flow rate declines slightly, and the sweep frequency first increases and then decreases, which is caused by the change of the main jet and the reduction of pressure difference.

(2) As CEW increases, the velocity of the main jet increases, resulting in a significant increase of the total flow rate. The sweep frequency reduces because of the further deflection distance of the main jet. And the pressure protuberance inside the cavity exit is the main reason for the change of the sweep angle.

(3) SR affects the volume of left and right shoulders, and then affects the development of the vortexes there, which leads to the change of spray characteristics.

(4) The total flow rate is slightly affected by IA and SR, but CEW is an effective measure to change total flow rate. Changing all the three parameters can alter the sweep angle in a wide range, but the change of impact angle is more regular. Variation of CEW can change the frequency in a higher range.

\section{Acknowledgments}

This study is supported by the National Nature Foundation (U1832809). The nozzle sample of utilized in this paper was provided by Jiangsu Riying Electronics Co., Ltd.

\section{Nomenclature}

IA impact angle

CEW cavity exit width

SR shoulder radius

\section{References}

[1] Cattafesta III, L. N., and Sheplak, M., 2011, "Actuators for active flow control," Annual Review of Fluid Mechanics, 43, pp. 247-272.

[2] Raman, G., and Cain, A., 2002, "Innovative actuators for active flow and noise control," Proceedings of the Institution of Mechanical Engineers, Part G: Journal of Aerospace Engineering, 216(6), pp. 303-324.

[3] Raghu,S., 2013, "Fluidic oscillators for flow control," Experiments in Fluids, 54(2), pp. 1-11.

[4] Gregory, J., and Tomac, M. N., 2013, "A review of fluidic oscillator development and application for flow control," 43rd AIAA fluid dynamics conference, p. 2474.

[5] Gregory, J. W., Sullivan, J. P., and Raghu, S., 2005, "Visualization of jet mixing in a fluidic oscillator," Journal of visualization, 8(2), pp. 169-176.

[6] Bidadi, S., Heister, S. D., and Matsutomi, Y., 2011, "Computational and experimental study of jet interaction fluidic injectors," Atomization and Sprays, 21(2).

[7] Tomac, M. N., and Gregory, J., 2012, "Frequency studies and scaling effects of jet interaction in a feedback-free fluidic oscillator," 50th AIAA Aerospace Sciences Meeting including the New Horizons Forum and Aerospace Exposition, p. 1248.

[8] Jhaveri, V., DeSalvo, M., Glezer, A., and Colton, J., 2019, "Effects of manufacturing parameters on performance of fluidic oscillators for aerodynamic flow control," Proceedings of the Institution of Mechanical Engineers, Part G: Journal of Aerospace Engineering, 233(10), pp. 3603-3611. 